\title{
Entanglement of Superposition and Superposition of Entanglement
}

\author{
Open Mathematics Collaboration*广
}

August 7, 2020

\begin{abstract}
We consider that the superposition of space is given by the Bell states and that those states are in superposition themselves.
\end{abstract}

keywords: entanglement, superposition, Bell states, quantum information

The most updated version of this paper is available at https://osf.io/zjdrm/download

\section{Introduction}

1. [1]

2. This article is an example/application of [2].

*All authors with their affiliations appear at the end of this paper.

†Corresponding author: mplobo@uft.edu.br | Join the Open Mathematics Collaboration 


\section{The electron}

3. Suppose that an electron is in a superposition of space from $x=0$ and $x=a$.

4. Let $A, B, C, D, E, F, G, \ldots$ be points between $x=0$ to $x=a$.

\section{Entanglement of superposition}

5. Conjecture 1: Two points in space are entangled.

6. $|A B\rangle=a|10\rangle_{A B}+b|01\rangle_{A B}$

7. $|10\rangle_{A B}=$ the electron collapsed at $A$

8. $|01\rangle_{A B}=$ the electron collapsed at $B$

9. The meaning of the first (second) ket is the presence (1) or absence (0) of the electron at a specific point in space.

\section{Superposition of entanglement}

10. Conjecture 2: All pairs of entangled spatial points are in a quantum superposition.

11. The quantum state of the electron, according to (5) and (10), is then given by

$$
\begin{aligned}
|\Psi\rangle & =\alpha_{1}|A B\rangle_{A B}+\alpha_{2}|A C\rangle_{A C}+\alpha_{3}|A D\rangle_{A D}+\ldots+\beta_{1}|B C\rangle_{B C}+ \\
& +\beta_{2}|B D\rangle_{B D}+\ldots+\gamma_{1}|C D\rangle_{C D}+\ldots
\end{aligned}
$$

\section{Discussion}

12. According to our approach, the mathematical definition of [2] is given by

$$
|\Psi\rangle=x_{1}|1000 \ldots 0\rangle+x_{2}|0100 \ldots 0\rangle+x_{3}|0010 \ldots 0\rangle+\ldots
$$




\section{Final Remarks}

13. We presented two conjectures stating that two points in space are entangled and also that all pairs of entanglement are in a quantum superposition.

14. The quantum nature of the interplay between objects and spacetime is somewhere between (5) and (12).

\section{Open Invitation}

Review, add content, and co-author this paper $[3,4]$.

Join the Open Quantum Collaboration (https://bit.ly/ojmp-slack). Send your contribution to mplobo@uft.edu.br.

\section{Open Science}

The latex file for this paper together with other supplementary files are available [5].

\section{Ethical conduct of research}

This original work was pre-registered under the OSF Preprints [6], please cite it accordingly [7]. This will ensure that researches are conducted with integrity and intellectual honesty at all times and by all means.

\section{Acknowledgement}

+ Center for Open Science https://cos.io 


\section{+ Open Science Framework}

https://osf.io

\section{References}

[1] Susskind, Leonard, and Art Friedman. Quantum mechanics: the theoretical minimum. Basic Books, 2014.

[2] Lobo, Matheus P. "Quantum Superposition as Entanglement." OSF Preprints, 25 Dec. 2019. https://doi.org/10.31219/osf.io/m2ajq

[3] Lobo, Matheus P. "Microarticles." OSF Preprints, 28 Oct. 2019. https://doi.org/10.31219/osf.io/ejrct

[4] Lobo, Matheus P. "Simple Guidelines for Authors: Open Journal of Mathematics and Physics." OSF Preprints, 15 Nov. 2019. https://doi.org/10.31219/osf.io/fk836

[5] Lobo, Matheus P. "Open Journal of Mathematics and Physics (OJMP)." OSF, 21 Apr. 2020. https://doi.org/10.17605/osf.io/6hzyp

[6] COS. Open Science Framework. https://osf.io

[7] Lobo, Matheus P. "Entanglement of Superposition and Superposition of Entanglement." OSF Preprints, 13 July 2020. https://doi.org/10.31219/osf.io/zjdrm

\section{The Open Quantum Collaboration}

Matheus Pereira Lobo (lead author, mplobo@uft.edu.br) $)^{1,2}$ https://orcid.org/0000-0003-4554-1372

${ }^{1}$ Federal University of Tocantins (Brazil)

${ }^{2}$ Universidade Aberta (UAb, Portugal) 